\title{
Double Dissociation of the Roles of Metabotropic Glutamate Receptor 5 and Oxytocin Receptor in Discrete Social Behaviors
}

\author{
Ivana Mesic', Yomayra F Guzman', Anita L Guedea', Vladimir Jovasevic', Kevin A Corcoran', \\ Katherine Leaderbrand', Katsuhiko Nishimori ${ }^{2}$, Anis Contractor ${ }^{3}$ and Jelena Radulovic*, \\ 'Department of Psychiatry and Behavioral Sciences, Northwestern University, Feinberg School of Medicine, Chicago, IL, USA; ${ }^{2}$ Department of \\ Molecular and Cell Biology, Graduate School of Agricultural Science, Tohoku University, Miyagi, Japan; ${ }^{3}$ Department of Physiology and Behavioral \\ Sciences, Northwestern University, Feinberg School of Medicine, Chicago, IL, USA
}

\begin{abstract}
Social interactions in vertebrates are complex phenomena based on affective and cognitive processes. Multiple brain regions and neurotransmitter systems are involved in the expression of social behaviors, but their individual roles in specific aspects of social interactions are not well understood. Here we investigated how Gq-protein-coupled metabotropic glutamate receptor 5 (mGluR5) and oxytocin receptor (Oxtr) affect social affiliation and social memory. We used conditional genetic approaches in which the genes coding for these receptors were knocked out in the lateral septum by infusion of recombinant adeno-associated viral vectors containing Cre recombinase (AAV-Cre). Social behavior was assessed 2 weeks later using a three-chamber paradigm for sociability and preference for social novelty. Septal deletion of mGluR5 abolished sociability while leaving preference for social novelty intact. In contrast, deletion of Oxtr did not affect sociability but significantly impaired preference for social novelty. Nonsocial behaviors or memories, including novel object recognition or fear conditioning, were not affected by these genetic manipulations. Immunohistochemical analyses of the distribution of mGluR5 and Oxtr revealed non-overlapping localization of these receptors within the lateral septum, suggesting that not only different neurotransmitters but also different neuronal types contribute to sociability versus preference for social novelty. Our findings identify highly specialized roles of lateral septal mGluR5 and Oxtr in the the regulation of discrete social behaviors, and suggest that deficits in social interactions, which accompany many mental illnesses, would benefit from comprehensive treatments targeting different components of social functioning.

Neuropsychopharmacology (20I5) 40, 2337-2346; doi:I0.I038/npp.20I5.8I; published online 22 April 2015
\end{abstract}

\section{INTRODUCTION}

Interactions among conspecifics constitute an important basis for the organization of life and provide many health benefits to social species. Deficits in social behavior are linked to developmental abnormalities in children, such as autism spectrum disorders (ASD), and can also occur later in life as a sign of major psychiatric illnesses such as schizophrenia or major depression (Crespi and Badcock, 2008). Understanding the mechanisms of social behavior therefore has important implications for mental health.

Several neurotransmitter systems have been causally linked to the expression of social behavior, including those acting via major excitatory and inhibitory ion channel receptors

*Correspondence: Dr J Radulovic, Department of Psychiatry and Behavioral Sciences, Feinberg School of Medicine, Northwestern University, 303 East Chicago Avenue, Ward 13-130, Chicago, IL 606 II, USA, Tel: + I 312503 4627, Fax: + I 3125030466 ,

E-mail: j-radulovic@northwestern.edu

Received 7 January 20 I 5; revised 23 February 20 I5; accepted I5 March 2015; accepted article preview online 3I March 2015
(Coghlan et al, 2012; Yang and Chang, 2014), as well as those acting via G-protein-coupled receptors (Caldu and Dreher, 2007; Kiser et al, 2012). Two classes of Gq-protein-coupled receptors, metabotropic glutamate receptor 5 (mGluR5) and oxytocin receptor (Oxtr), are of particular interest: both have been found to contribute to social, affective, and stress-related behavior (Panksepp, 1992; Carter, 1998; Yap et al, 2005; Koros et al, 2007) and have been implicated as potential treatment targets for ASD symptoms (Insel et al, 1999; Dolen et al, 2010).

Abnormal mGluR5 function has been suggested as a contributing factor to ASD; thus, mGluR5 manipulations has been extensively studied by using systemic pharmacological manipulations in genetic mouse models of social deficits (Dolen et al, 2010; Carlson, 2012). Unfortunately, results from these studies have been inconsistent, depending on the genetic abnormality and behavioral phenotype. This is further complicated by findings that both positive (Clifton et al, 2013) and negative (Silverman et al, 2012) allosteric modulators of mGluR5 confer model- and phenotypespecific behavioral benefits. For example, impaired social 
novelty discrimination induced by neonatal treatment with phencyclidine is improved by activation of mGluR5 with positive allosteric modulators (Clifton et al, 2013). On the other hand, deficits of sociability in BTBR mice were improved by negative allosteric modulators of mGluR5 (Silverman et al, 2012). Interestingly, the role of mGluR5 in the social behavior of normal, genetically intact mice has also provided controversial evidence, with pharmacological approaches yielding strain-dependent effects. Antagonism of mGluR5 in Balb/c mice did not affect time spent in a chamber with a juvenile but it enhanced sniffing, whereas the same manipulation in Swiss Webster mice enhanced time spent in the chamber but reduced sniffing (Burket et al, 2011). Overall, the role of mGluR5 in social behavior has not yet been clarified, possibly because of the limited specificity of pharmacological approaches, reliance on acute treatments, or use of systemic manipulations.

Unlike mGluR5, oxytocin has traditionally been viewed as 'the' social hormone and a number of pharmacological manipulations of Oxtr has implicated this system in virtually every aspect of social functioning (Ross and Young, 2009). Surprisingly, genetic deletion of oxytocin or Oxtr has produced specific deficits of social memory while leaving other social functions intact (Ferguson et al, 2000; Crawley et al, 2007). However, even in genetic models, the data have not been consistent, and more general deficits have also been reported (Sala et al, 2013). It is not yet clear whether these discrepancies are due to insufficient specificity of pharmacological manipulations, compensatory mechanisms in constitutive Oxtr knockouts, or to the complex and possibly opposing contributions of different brain areas and circuits to social behaviors.

We attempted to establish the roles of mGluR5 and Oxtr on different aspects of social behavior in a comparative study, using conditional and region-specific genetic manipulations of these receptors. We selected this approach to achieve highly specific manipulations of each receptor while minimizing the possibility of compensation. We focused on the lateral septum because this area contains some of the highest brain levels of Oxtr (Lee et al, 2008) and mGluR5 (Shigemoto et al, 1993; Romano et al, 1995) and is therefore a likely target for their actions. In addition, the lateral septum has a well-established role in social behavior and it is directly connected (Risold and Swanson, 1997) to other key brain areas regulating social interactions, such as CA2 hippocampus (Hitti and Siegelbaum, 2014) and dopaminergic circuits (Gunaydin et al, 2014). To examine the specificity of Oxtr and mGluR5 in social versus nonsocial behavior, we also tested the mice in novel object recognition and fear conditioning paradigms. Our findings demonstrate highly specific roles of septal mGluR5 and Oxtr in social behavior, as indicated by a double dissociation of their effects on sociability and social novelty.

\section{MATERIALS AND METHODS}

\section{Animals}

Nine-week-old male C57BL/6J mice with floxed mGluR5 (Xu, 2009, p 669) or Oxtr (Nishimori, 2008, p 1830) were backcrossed for over nine generations into wild-type C57BL/6J females (Jackson) and subsequently maintained by homozygous breeding in Northwestern University's animal facility. After weaning, the mice were individually housed on a $12 \mathrm{~h}$ light/ dark cycle (lights on at 0700 hours), and allowed ad libitum access to food and water. Nine- to eleven-week-old mice were used for the experiments. Wild-type C57BL/6N male mice (Harlan) of the same age served as the stimulus mice. One week before the beginning of the experiments, the mice were transferred to our satellite facility, which provides similar environmental conditions. In the facility, the mice were housed in laminar flow cabinets (Scanbur, Sollentuna, Sweden) adjacent to the behavioral testing equipment to avoid transport between housing and testing. Separate groups of mice were used for each behavioral paradigm. All procedures were approved by Northwestern University's Animal Care and Use Committee in compliance with National Institutes of Health standards.

\section{Surgery and Cannulation}

Double guide cannulas (Plastics One) were implanted in the lateral septum as described previously (Guzman et al, 2013). Briefly, mice were anesthetized with $1.2 \%$ tribromoethanol (Avertin) and implanted with bilateral cannulas (26-gauge outer and 28-gauge inner diameter, $1 \mathrm{~mm}$ center-to-center distance) using a digital stereotaxic apparatus (Kopf Instruments). The coordinates used to target lateral septum were $0.2 \mathrm{~mm}$ anterior $\pm 0.5 \mathrm{~mm}$ lateral, and $3.0 \mathrm{~mm}$ ventral to bregma. Mice were allowed 1 week to recover before virus injections. Only mice with correct cannula placements, as determined at the end of the experiments with histological examination of brain tissue, were included in data analyses.

\section{Infusion of Viral Vectors}

The viral vectors AAV-GFP-Cre (AAV-Cre; driven by a synapsin promoter and obtained from Pavel Osten) or AAVGFP (driven by a synapsin promoter, University of Pennsylvania Viral Vector Core) were infused intraseptally over $2 \mathrm{~min}(0.1 \mu \mathrm{l} / \mathrm{min})$ under light isoflurane anesthesia. All viruses had a titer of $1-2 \times 10^{12}$ particles per $\mathrm{ml}$ and were of the AAV2 serotypes. Before any behavioral training, viruses incubated for 14 days to allow for significant knockdown. After the end of each experiment, all brains were collected for histological verification of cannula placements or immunohistochemical or quantitative PCR (qPCR) validation of genetic knockdown. We demonstrated previously that the AAV-GFP and AAV-Cre do not exert toxic effects on lateral septal neurons, and that AAV-Cre did not affect memory-related or other behaviors of wild-type mice (Guzman et al, 2013). On this basis, and to minimize confounds of genetic factors (eg with comparisons of floxed versus wild-type mice), all experiments were performed with floxed mice receiving either AAV-GFP or AAV-Cre.

\section{Social Interaction Paradigm}

To assess sociability and social novelty, we used a threecompartment social behavior chamber (Moy et al, 2004; Sankoorikal et al, 2006). The chamber was a glass rectangular box $(76.2 \mathrm{~cm}$ long $\times 30.5 \mathrm{~cm}$ wide $\times 30.5 \mathrm{~cm}$ tall $)$ divided into three compartments with a mesh cylinder ( $11.4 \mathrm{~cm}$ diameter) placed in each side compartment. The mesh cylinder allowed 
for air exchange and for auditory, visual, and olfactory interaction, but prevented fighting, between experimental and stimulus mice. Testing consisted of a 5-min habituation phase, in which no stimuli were present; a 5-min sociability test, in which a stimulus mouse and an inanimate object (toy mouse) were present in separate chambers; and a 5-min preference for social novelty test, in which the toy mouse was replaced by a novel mouse. Animals' position and motion were tracked by a top-mounted video camera connected to a computer and a DVD recorder and analyzed using Videomot II tracking software (TSE Systems). For analysis, time spent in each compartment during each phase of the test and time spent in close proximity (within $4 \mathrm{~cm}$ ) of the stimulus mice during the test phase were calculated and expressed as percent of the total test time $(5 \mathrm{~min})$. Because these parameters yielded similar results, we present only the percent of time spent in each compartment relative to the total test time so that the habituation data (without a stimulus) can also be compared. Sociability was assessed by the time spent with a mouse versus an object, whereas preference for social novelty was assessed by the time spent with a new versus a familiar mouse, and each was presented as percent of the total test time.

\section{Novel Object Recognition Paradigm}

The novel object recognition paradigm consisted of an identical apparatus and involved a similar procedure as described above, except that instead of conspecifics, mice were exposed to different objects as described previously (Gao et al, 2011). Briefly, after a 5-min habituation period, the mice were exposed for $5 \mathrm{~min}$ to a cube in one, and a bottle in the other side compartment. Subsequently, one of the objects was randomly replaced with a novel object (a tape roll), and the time spent in the chamber of the novel versus familiar object was calculated relative to total test time.

\section{Fear Conditioning Paradigm}

Fear conditioning took place in a $35 \times 20 \times 20 \mathrm{~cm}^{3}$ Plexiglas chamber with a stainless-steel rod floor $(4 \mathrm{~mm}$ diameter, $0.9 \mathrm{~cm}$ center to center) located within a sound-attenuating cabinet with black inner walls (TSE Systems). The box was cleaned after each mouse with $70 \%$ ethanol. Mice were placed in the chamber and 3 min later presented with a footshock ( $2 \mathrm{~s}, 0.7 \mathrm{~mA}$, constant current). Mice were tested for fear to the conditioning context $24 \mathrm{~h}$ later by returning them to this chamber for $3 \mathrm{~min}$ tests. Freezing was scored every $5 \mathrm{~s}$ by a trained observer blind to the experimental conditions, and expressed as the percentage of total number of observations (Guzman et al, 2013).

\section{RNA Extraction and qPCR for Determination of Oxtr mRNA Levels}

Mice were injected intraseptally with methylene blue to visualize the viral injection site and then killed by cervical dislocation. Brains were dissected and the lateral septum was collected using a brain matrix. Tissue was homogenized in lysis buffer with $\beta$-mercaptoethanol and frozen in liquid nitrogen. RNA was extracted using miRCURY Total RNA Isolation Kit (Exiqon), reversely transcribed, and subjected to real-time PCR using SYBR Green master mix (Applied Biosystems) and primers for Oxtr or mouse hypoxanthine phosphoribosyl transferase 1 ( $m$ Hprt 1 ) as an internal control (Tronson et al, 2009). For Oxtr, the forward primer was 5'-GGAGCGTCTGGGACGTCAAT-3' and the reverse primer was $5^{\prime}$-AGGAAGCGCTGCACGAGTT-3'. For $m H$ prt 1 , the forward primer was 5'-GGGCTTACCTCACTGCTTTC-3' and reverse 5'-TCTCCACCAATAACTTTTATGTCC-3'. The level of Oxtr expression in Oxtr flox/flox mice injected with $\mathrm{rAAV}$ Cre was normalized to mHprt 1 and shown relative to the rAAV-GFP control.

\section{Immunoblot}

Septal tissue was collected around the tips of the cannula and lysed in modified radioimmunoprecipitation buffer $(20 \mathrm{mM}$ Tris- $\mathrm{HCl}$ ( $\mathrm{pH}$ 7.5), $150 \mathrm{mM} \mathrm{NaCl}, 1 \mathrm{mM} \mathrm{Na}{ }_{2}$ EDTA, $1 \mathrm{mM}$ EGTA, $1 \% \mathrm{NP}-40,1 \mathrm{mM} \mathrm{Na} \mathrm{VO}_{4}$ ) containing a protease inhibitor cocktail (Pierce), incubated for $15 \mathrm{~min}$ on ice, and centrifuged for $15 \mathrm{~min}(15000 \mathrm{~g})$ at $4{ }^{\circ} \mathrm{C}$. Samples were subjected to SDS-PAGE (10 $\mu \mathrm{g}$ per well) and transferred to polyvinylidene difluoride membranes (Millipore). Membranes were blocked with I-block (Tropix), incubated with primary antibody overnight at $4{ }^{\circ} \mathrm{C}$, and with secondary antibody conjugated to alkaline phosphatase for $1 \mathrm{~h}$ at room temperature, followed by incubations with the chemiluminescent enhancer Nitroblock II and chemiluminescent substrate CDP star (Tropix). Bands were visualized after exposing the membranes to X-ray films. Primary antibodies used were against $\alpha$-tubulin, 1:2000 (mouse; Sigma), and anti-mGluR5, 1:6000 (rabbit; Millipore), as shown previously (Tronson et al, 2010).

\section{Immunohistochemistry}

Brains were fixed with 4\% PFA, dehydrated in 30\% sucrose, and stained using the Vectastain system (Vector), as described previously (Guzman et al, 2013). Immunostaining with mouse anti-GFP (1:6000; Abcam) was visualized with fluorescein isothiocyanate, whereas rabbit anti-mGluR5 (1:16000; Millipore) was visualized with Coumarin. Slices were mounted in Vectashield (Vector) and observed with a confocal laser scanning microscope (Olympus Fluoview FV10i) for double labeling at $\times 40$. Septal sections were analyzed for double labeling at the coordinates (Paxinos and Franklin, 2004) AP +0.14 to +0.38 . Quantification of mGluR5 signals was performed using the ImageJ software, by analyzing the density of mGluR5 signals or determining the number of Venus-positive neurons in the dorsal and lateral septum.

\section{Statistical Analysis}

We used 7-10 mice per experimental group for the behavioral studies. For immunohistochemical, qPCR, and immunoblot assays, we used biological replicates of 4-6 samples per group. This sample size gave us statistical power of $80 \%$ to detect knockdown effects. All data showed normal distribution and were analyzed by repeated-measures ANOVA with factors genotype and test condition (sociability versus social novelty). Two-tailed Student's $t$-test was used to determine 
within-group side preference. Data are presented as mean \pm SEM.

\section{RESULTS}

\section{Conditional Knockdown of mGluR5 and Oxtr by} rAAV-Cre

Infusion of rAAV-Cre resulted in a conditional mGluR5 knockdown in the lateral septum in $\mathrm{mGluR} 5^{\text {flox/flox }}$ mice, with an average decrease of $\sim 75 \%$ of mGluR5 protein levels when compared with the rAAV-GFP controls (Figure 1a; $t(9)=8.081, \quad P<0.001, \quad N=5$ per group). Validation of cannula placements by histological analysis confirmed the septal infusion sites (Figure 1b). Because specific Oxtr antibodies were not available, Oxtr knockdown in Oxtr ${ }^{\text {flox/flox }}$ was validated with qPCR. Similar to our earlier observations (Tronson et al, 2009; Guzman et al, 2014), infusion of rAAVCre resulted in a conditional Oxtr knockdown in the lateral septum, with an average decrease of $\sim 70 \%$ of Oxtr mRNA when compared with the rAAV-GFP control (Figure 1c; $t(7)=7.65, P<0.001, n=4$ per group). Cannula placements from these experiments are depicted in Figure 1d. These studies showed that rAAV-Cre caused a significant knockdown of mGluR5 and Oxtr in their corresponding floxed mouse lines.

\section{mGluR5 Knockdown in the Lateral Septum Disrupts} Sociability but not Preference for Social Novelty

mGluR $5^{\text {flox/flox }}$ mice infused with rAAV-GFP or rAAV-Cre did not show innate preference for any side of the chamber during habituation (Figure 2a; rAAV-GFP: $t(1,17)=0.118$, $P=0.903$; rAAV-Cre: $t(1,17)=0.112, P=0.913, n=9$ per group). On tests of sociability and social novelty, repeatedmeasures ANOVA found significant effects of test $(\mathrm{F}(1,16)=107.66, \quad P<0.001), \quad$ group $\quad(\mathrm{F}(1,16)=11.21$, $P<0.01)$, and test $\times$ genotype $(\mathrm{F}(1,16)=21.83, P<0.001)$ interaction. Repeated contrary to rAAV-GFP controls, who displayed a significant preference for the social versus the nonsocial stimulus (Figure $2 \mathrm{~b} ; t(1,17)=3.119, P<0.05)$ ), rAAV-Cre group similarly explored the social and nonsocial stimulus $(\mathrm{t}(1,17)=0.219, P=0.830)$. These findings demonstrate decreased sociability in mGluR5 knockdown mice. Despite this deficit, the mice showed preserved preference for social novelty (Figure 2c) and spent increased time exploring the novel versus familiar stimulus mice (rAAVGFP: $t(1,17)=3.43, \quad P<0.05$; rAAV-Cre: $t(1,17)=2.961$, $P<0.05)$. These findings revealed a specific deficit of sociability induced by mGluR5 knockdown.

\section{Oxtr Knockdown in the Lateral Septum Disrupts Preference for Social Novelty but not Sociability}

Similar to $\mathrm{mGluR} 5^{\text {flox/flox }}$ mice, Oxtr ${ }^{\text {flox/flox }}$ mice infused with rAAV-GFP or rAAV-Cre equally explored the different sides of the chamber $(t(1,17)=0.149, P=0.955, n=9$ per group) during habituation (Figure 3a). On tests of sociability and social novelty, repeated-measures ANOVA found significant effects of test $(\mathrm{F}(1,16)=76.01, P<0.001)$, group $(\mathrm{F}(1,16)=$ $8.246, \quad P<0.05)$, and test $\times$ genotype $(\mathrm{F}(1,16)=24.68$, $P<0.001)$ interaction. Lateral septal Oxtr knockdown did a
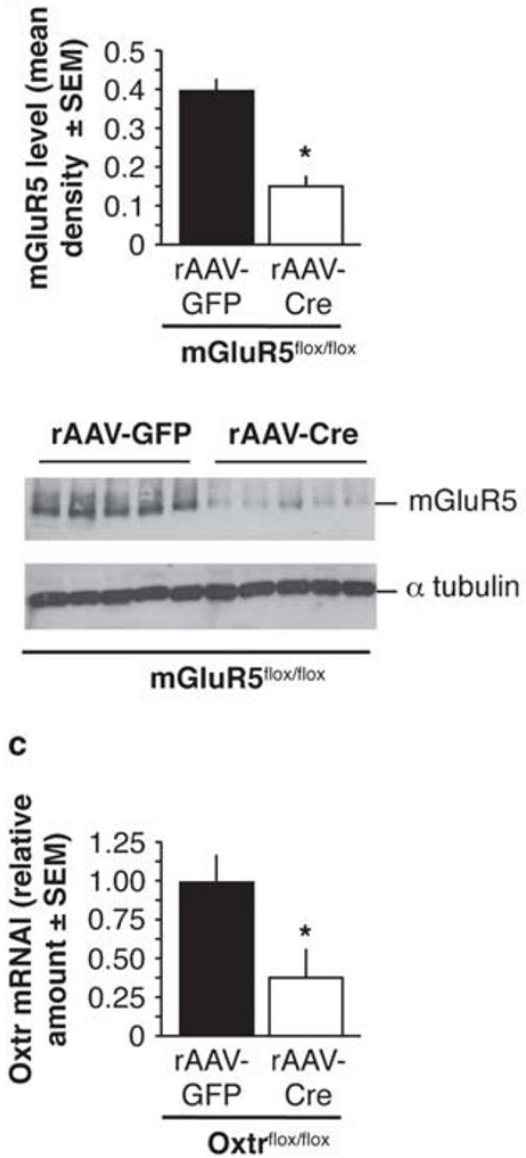

Figure I Conditional knockdown of mGluR5 and Oxtr in the lateral septum. (a) Protein levels of mGluR5 in septal lysates of mGluR5 ${ }^{\text {flox/flox }}$ mice injected with rAAV-GFP or rAAV-Cre. The immunoblot is shown below. (b) Cannula placement sites for mice used in the mGluR5 knockdown experiments. An example of GFP signals in the lateral septum is illustrated below. (c) Oxtr mRNA levels in septi of Oxtr flox/flox mice injected with rAAV-GFP or rAAV-Cre. (d) Cannula placement sites for mice used in the Oxtr knockdown experiments. Statistically significant differences: ${ }^{*} P<0.001$, rAAV-Cre versus rAAV-GFP controls.

not affect sociability (Figure 3b), as revealed by significantly more time spent with the social versus nonsocial stimulus in both rAAV-GFP $(t(1,17)=3.55, P<0.05)$ and rAAV-Cre groups $(\mathrm{t}(1,17)=3.24, P<0.05)$. However, preference for social novelty was significantly disrupted (Figure $3 \mathrm{c}$ ), as revealed by the finding that $\mathrm{rAAV}$-Cre mice similarly explored the novel and familiar stimulus mouse $(t(1,17)=0.186, P=0.645)$, whereas rAAV-GFP controls showed significant preference for the novel stimulus mouse $(t(1,17)=4.27, P<0.01)$. Thus, contrary to the mGluR5 knockdown, Oxtr knockdown did not affect sociability but selectively impaired preference for social novelty.

\section{mGluR5 or Oxtr Knockdown in the Lateral Septum do not Affect Novel Object Recognition Memory}

We used the novel object recognition task to examine whether the observed deficits were specific for social behavior or also extend to nonsocial stimuli. All objects were selected based 
a

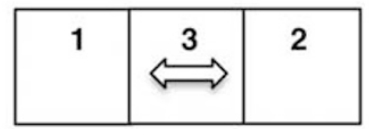

b

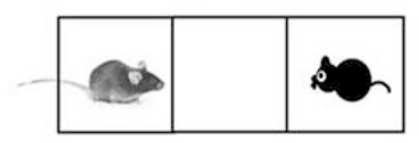

C

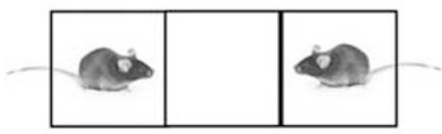

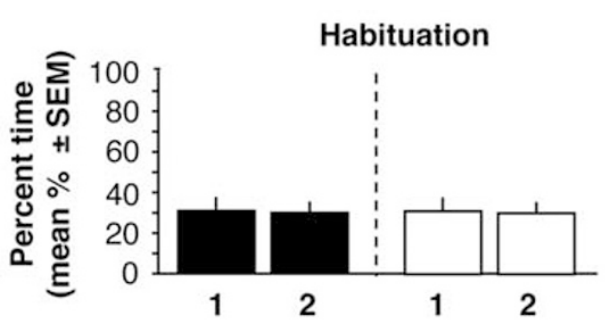

Sociability
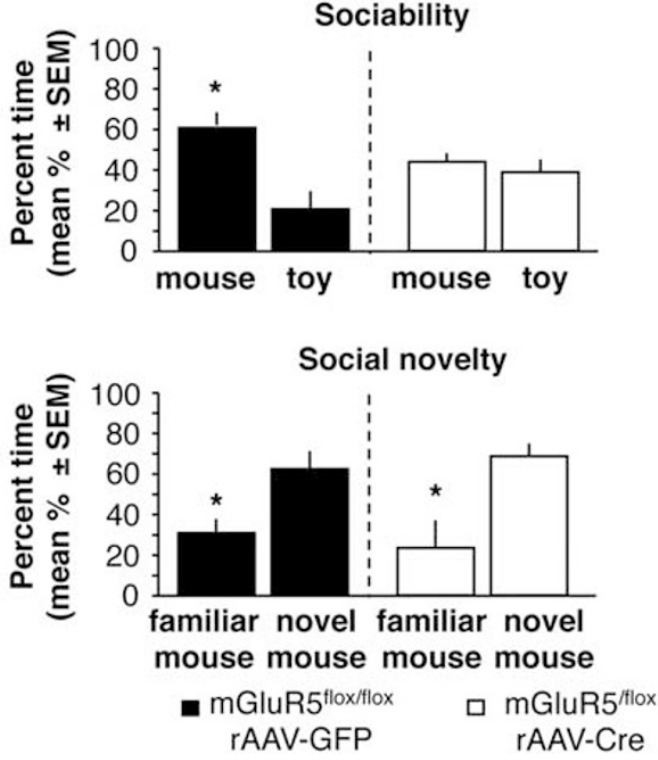

Figure 2 Effect of septal mGluR5 knockdown on sociability and social novelty. (a) Behavior recorded during habituation and expressed as \% time spent in the side compartments of the chamber. (b) Sociability, expressed as \% time spent with the conspecific when compared with the toy mouse. (c) Preference for social novelty, expressed as \% time spent with the novel when compared with the familiar mouse. Statistically significant differences: $* P<0.05$, versus corresponding control (toy mouse or novel mouse). Note: the schemes on the left illustrate the general design; however, during experiments the side was counterbalanced.

on their similar preference by the mice (Gao et al, 2011). Accordingly, mice of each group expressed similar interest in the objects during the first exposure, for mGlur $5^{\text {flox/flox }}$ (Figure $4 \mathrm{~b} ; \mathrm{F}(3,28)=0.225, P=0.72, n=7-8$ ) and Oxtr ${ }^{\text {flox/flox }}$ mice (Figure 4e; $\mathrm{F}(3,27)=0.134, P=0.682, n=7-8)$. On tests of exposure to a novel versus familiar object, repeatedmeasures ANOVA performed on mGluR5 knockdown found significant effects of test $(\mathrm{F}(1,13)=150.08, P<0.001)$ but not genotype $(\mathrm{F}(1,16)=0.851, P=0.373)$ or test $\times$ genotype $(\mathrm{F}(1,16)=2.98, P=0.104)$ interaction. Similar results were obtained for the Oxtr knockdown, with significant effects of test $(\mathrm{F}(1,13)=123.22, \quad P<0.001)$ but not group $(\mathrm{F}(1,13)=0.714, \quad P=0.325)$ or test $\times$ genotype $(\mathrm{F}(1,13)=$ 2.44, $P=0.113$ ) interaction. An overall one-way ANOVA on the test day revealed that both mGluR5 and Oxtr knockdown mice, as well as controls, recognized the novel object in the last phase of the paradigm, as revealed by a significantly increased time spent in the chamber of the novel when compared with the familiar object (Figure $4 \mathrm{c}$; $\mathrm{F}(3,26)=8.43, \quad P<0.01, \quad n=7-8)$ and $\mathrm{Oxtr}^{\text {flox/flox }}$ mice (Figure 4f; $\mathrm{F}(3,26)=7.92, P<0.01, n=7-8$ ). These findings show that interest in and recognition of inanimate objects was intact in mGluR5 and Oxtr knockdown mice, and thus their deficits were restricted to social behavior.

\section{mGluR5 or Oxtr Knockdown in the Lateral Septum do not Affect Fear Conditioning}

In addition to social behavior, the lateral septum mediates fear- and anxiety-related behaviors (Gray and McNaughton, 2000). Both mGluR5 and Oxtr have been implicated in the modulation of contextual fear by social or nonsocial stress (Tronson et al, 2010; Guzman et al, 2013), thus we next examined whether the septal knockdown of these receptors affected contextual fear conditioning. The results did not reveal any differences in freezing behavior between mGluR5 (Figure 5a; $t(15)=0.223, P=0.85, N=8$ per group) or Oxtr knockdown mice (Figure $5 b ; t(15)=0.257, P=0.88, N=8$ per group) and their corresponding controls, suggesting that neurotransmitter systems other than mGluR5 or Oxtr regulate fear-motivated learning via the lateral septum.

\section{mGluR5 and Oxtr are Expressed in Different Neuronal Populations of the Lateral Septum}

The double dissociation of the effects of mGluR5 and Oxtr on sociability and preference for social novelty could be due to actions of these receptors on the same neuronal population (but using different signaling pathways) or by 
a

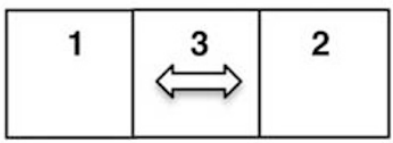

b
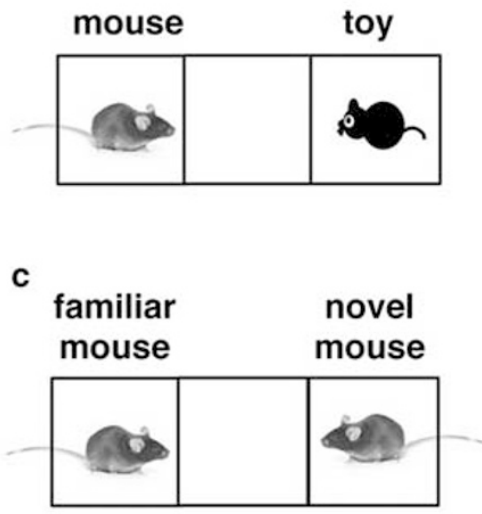
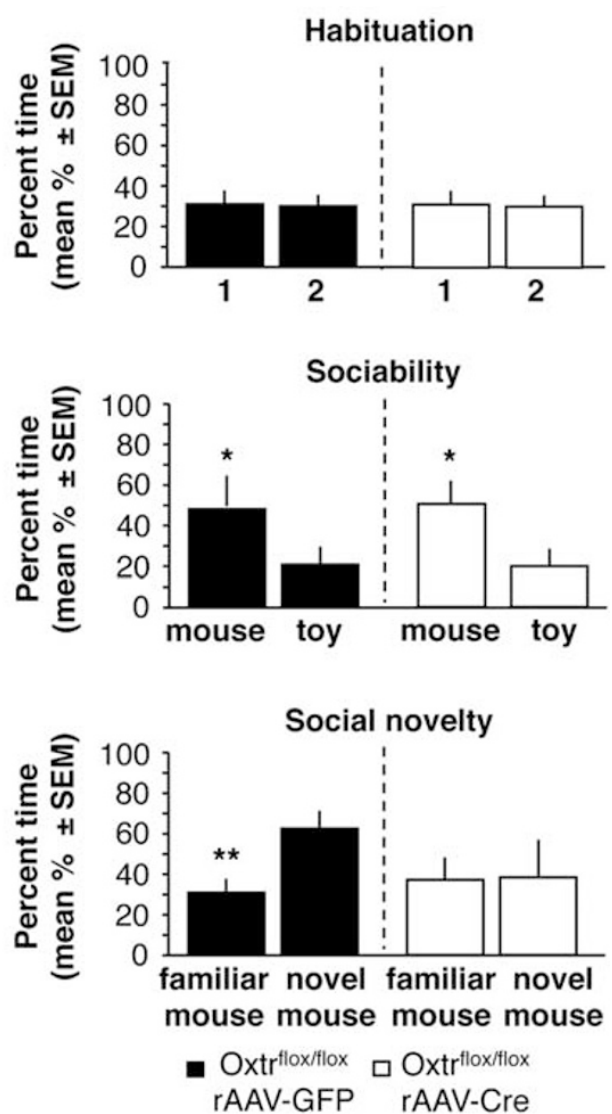

Figure 3 Effect of septal Oxtr knockdown on sociability and social novelty. (a) Behavior recorded during habituation and expressed as \% time spent in the side compartments of the chamber. (b) Sociability, expressed as \% time spent with the conspecific when compared with the toy mouse. (c) Preference for social novelty, expressed as \% time spent with the novel when compared with the familiar mouse. Statistically significant differences: * $P<0.05$, versus toy mouse; or novel mouse; ${ }^{*} * P<0.01$, versus novel mouse.

engaging different neuronal populations. To better understand the distribution of mGluR5 and Oxtr in the lateral septum, we performed a colocalization study. We first demonstrated that mGluR5 are abundant at the lateral septum (Figure 6a), at neuroanatomical coordinates showing strong Oxtr signals (Guzman et al, 2013). We next confirmed, using immunofluorescent labeling of Venus (green) in Venus/Oxtr reporter mice (Yoshida et al, 2009) and mGluR5 (blue) (Figure 6b), that both signals were the strongest in the caudal part of the lateral septum. However, whereas mGluR5 signals were most prominent in the dorsal and dorsolateral area, Oxtr-positive cells were predominantly found in the intermediate- and ventrolateral area. Our microscopic analyses did not reveal significant colocalization of mGluR5 and Oxtr (Figure 6c), given that mGluR5 signals were significantly higher in the dorsal versus lateral septum $(t(7)=8.45, P<0.001)$, whereas Venus signals were predominant in the lateral versus dorsal septum $(t(7)=7.96$, $P<0.001)$. This suggested that different neuronal populations of the lateral septum are engaged in the regulation of sociability and preference for social novelty.

\section{DISCUSSION}

We demonstrated that lateral septal mGluR5 and Oxtr has specific roles in the expression of social behaviors, with distinct contributions of mGluR5 and Oxtr to sociability and preference for social novelty. These findings suggest that rather than regulating social behavior in general, regional neurotransmitter systems mediated by G-protein-coupled receptors regulate different aspects of social functioning.

Research on social behavior was markedly boosted by its implications in ASD, and many genetic mouse models displaying abnormal social interactions have been used to explore potential therapeutics. One of the earliest models implicating mGluR5 in social behavior relevant for ASD is the fragile $\mathrm{X}$ syndrome model with Fmr1 mutant mice, in which enhanced mGluR5 activity has been linked to disease symptoms (Nakamoto et al, 2007). Systemic administration of the noncompetitive mGluR5 antagonist 2-methyl-6phenylethynyl-pyridine (MPEP) normalized seizures, prepulse inhibition, and dendritic spine densities (Yan et al, 2005; de Vrij et al, 2008). Genetic manipulations, however, did not support the link between enhanced mGluR5 activity and impairments of social behavior, as revealed by subtle, if any improvements of social behaviors in Fmrl mutant mice (Thomas et al, 2011). In a different model, the BTBR inbred mouse strain, MPEP improved stereotyped behavior without affecting sociability (Silverman et al, 2010), whereas a more specific mGluR5 antagonist, GRN-529, partially reversed the deficits of sociability (Silverman et al, 2012). Finally, other models yielded quite opposite findings, suggesting that activation of mGluR5 is needed for adequate social behavior. 

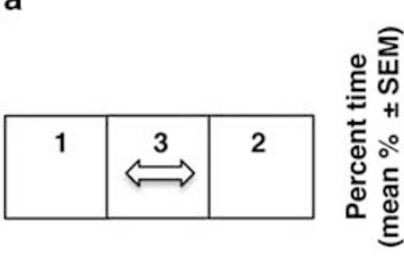

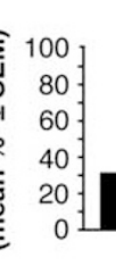

Habituation

b

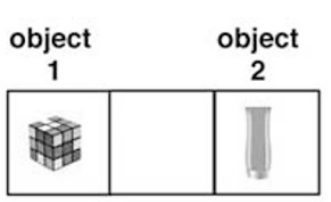

c

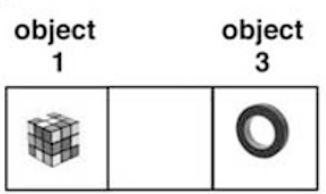

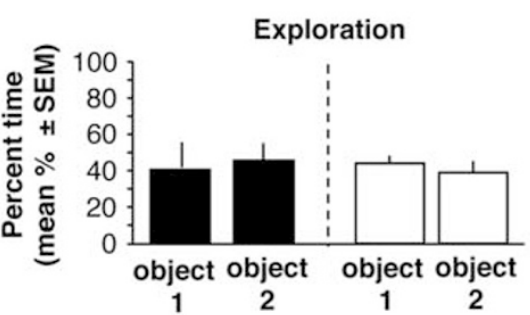

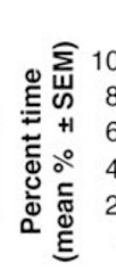

Novel object preference

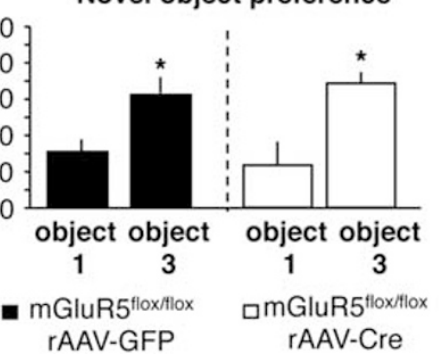

d

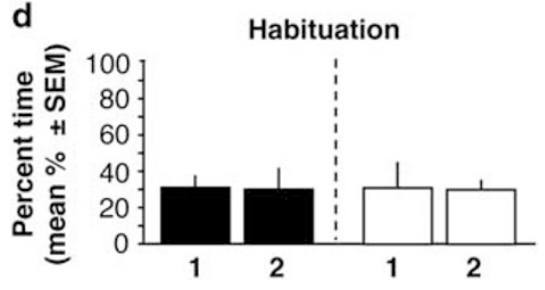

e

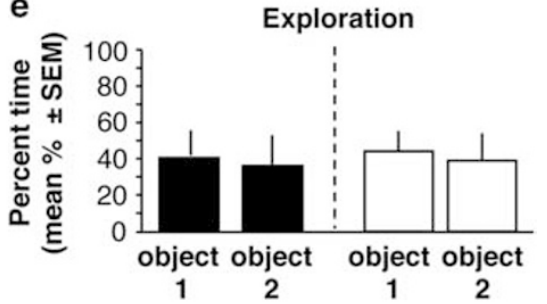

f

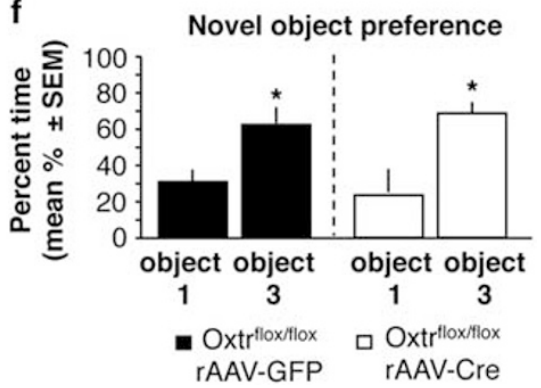

Figure 4 Effect of septal mGluR5 and Oxtr knockdown on novel object recognition. (a and b) Behavior of rAAV-GFP or rAAV-Cre-injected mGluR5 flox/llox mice during habituation and object exploration. (c) Preference for novel object, expressed as \% time spent with object 3 when compared with the familiar object I. ( $d$ and e) Behavior of rAAV-GFP or rAAV-Cre-injected Oxtr flox/flox mice during habituation and object exploration. ( $f$ ) Preference for novel object, expressed as \% time spent with object 3 when compared with the familiar object I. Statistically significant differences: *P $<0.0$ I, versus familiar object. As in Figures 2 and 3, the schematics depicts the general setup, but the object side was counterbalanced within a single experiment.

a

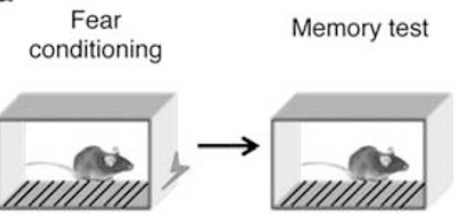

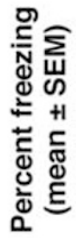

- mGluR5 $5^{\text {floxillox }}$ TAAV-GFP

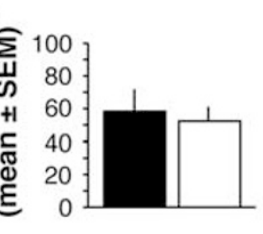

amGluR5 $5^{\text {floxillox }}$ b

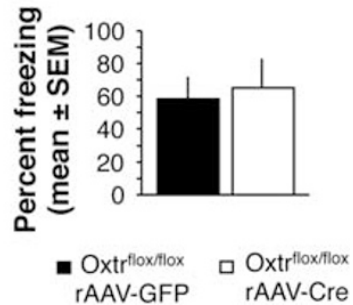

Figure 5 Effect of septal mGluR5 and Oxtr knockdown on contextual fear conditioning. (a) Behavior of rAAV-GFP or rAAV-Cre-injected mGluR5 flox/lox mice during a context memory test expressed as \% time spent freezing. (b) Behavior of rAAV-GFP or rAAV-Cre-injected Oxtrlox/flox mice during a context memory test expressed as \% time spent freezing.

In the ASD model of Shank2 knockout mice, a positive allosteric modulator of mGluR5 improved the social interaction deficits (Won et al, 2012). Similarly, in a developmental model of ASD, positive allosteric modulation of mGluR5 improved adult onset social deficits (Clifton et al, 2013). Unlike disease models, physiological studies of the role of mGluR5 in social behavior have been scarce, and have mainly relied on systemic injection of MPEP. This drug blocks $N$-methyl-D-aspartate (NMDA) receptors (O'Leary et al, 2000; Movsesyan et al, 2001) and monoamine oxidase-A (Cosford et al, 2003) in addition to mGluR5, which could have contributed to the inconsistent effects on sociability in Balb and Swiss-Webster mice (Burket et al, 2011). Our data are in agreement with findings obtained using the more specific mGluR5 antagonist, 3-[(2-methyl-4-thiazolyl) ethynyl] pyridine, MTEP, which impairs sociability both after acute and subchronic administration without affecting locomotor hyperactivity or stereotypy (Koros et al, 2007). Here we provide genetic evidence supporting an important and specific contribution of mGluR5 to sociability, and identify the lateral septum as the neuroanatomical target of this action. Taken together, these findings suggest that, 


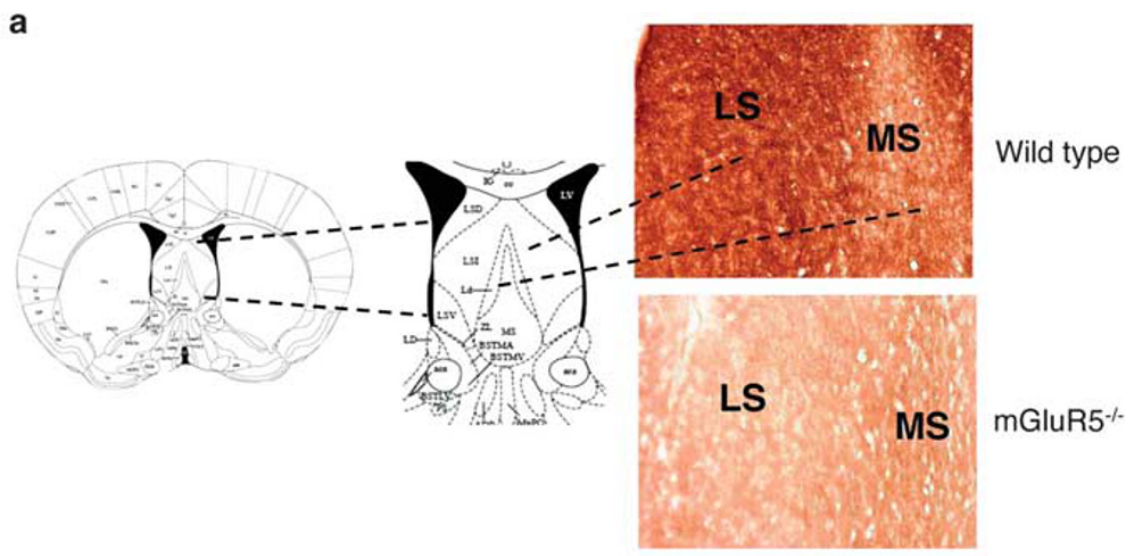

b

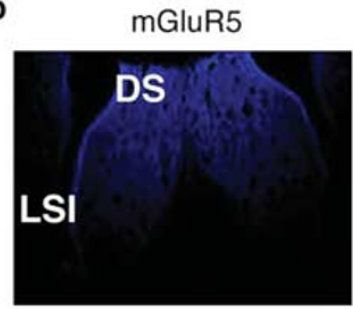

C

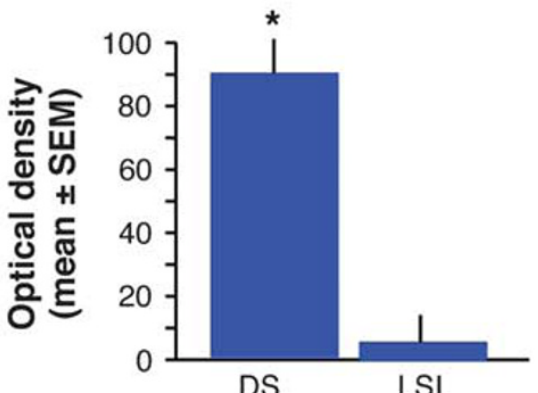

Venus

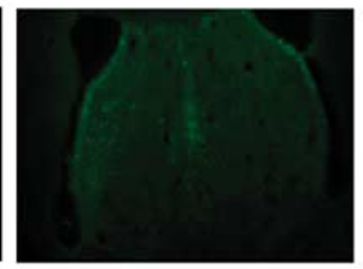

LSI

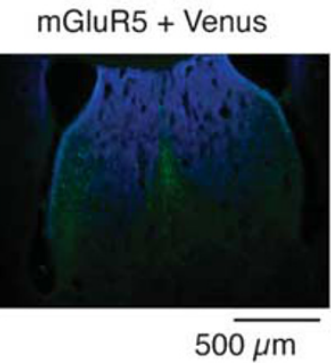

Venus

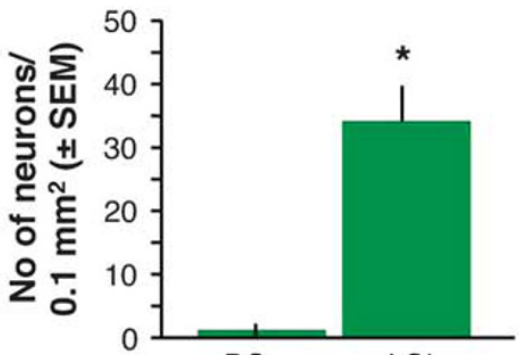

DS

LSI

Figure 6 Localization of mGluR5 and Oxtr in the lateral septum. (a) Validation of the specificity of anti-mGluR5 antibody in wild-type and mGluR5 ${ }^{-/-}$ mice. The schematics show the anatomical level of the images shown in the right panel. The antibody gave strong signals throughout the dorsomedial neuropil of the septum, whereas only background staining was noted in the medial septum. (b) Similar mGluR5 signals (blue) were detected in Venus reporter mice (left panel). Sections of these mice also showed Venus-positive neurons in intermediate (LSI) but not dorsal (DS) lateral septum (middle panel). The mGlu5 and Venus signals did not overlap (right panel). (c) Quantification of mGluR5 density and number of Venus-positive neurons in the DS and LSI. Statistically significant differences: $* P<0.00$ I, dorsal versus intermediate lateral septum.

unless the receptor is overactivated, antagonists and negative allosteric modulators of mGluR5 are more likely to worsen rather than improve deficits of sociability, whereas positive allosteric modulators might prove beneficial.

Contrary to mGluR5, the physiological role of oxytocin and Oxtr has been extensively investigated, with the majority of findings supporting a role in affiliative behavior and sociability (Neumann, 2008; Caldwell, 2012). Based on these findings, oxytocin has been linked to ASD (Insel et al, 1999) with promising outcomes in animal models (Teng et al, 2013). Surprisingly, approaches with constitutive genetic knockouts of oxytocin and Oxtr did not provide unequivocal support to this view. Oxytocin and Oxtr knockouts exhibited profound deficits of social memory (Ferguson et al, 2000; Lee et al, 2008); however, studies on sociability and preference for social novelty provided inconsistent data. One study reported no effects in two knockout lines (Crawley et al, 2007), whereas others reported deficits of preference for social novelty in knockouts (Lee et al, 2008; Pobbe et al, 2012) or deficits of both sociability and preference for social novelty in Oxtr+/ - mice (Sala et al, 2013). Compensation, in particular via the vasopressin system, could have contributed to the restricted behavioral phenotypes of genetic manipulations. Our findings, after inducible knockdown of Oxtr, fully support the specific role of Oxtr in social memory, as shown by intact sociability but impaired preference for social novelty, as well as social recognition, as shown earlier (Guzman et al, 2013).

Both sociability and preference for social novelty have been used to model the first of three core symptoms of ASDs: 
abnormal social interactions, impaired communication, and stereotyped behavior. However, these phenotypes engage different processes: sociability primarily reflects the motivation to interact with conspecifics, whereas preference for social novelty can be viewed as a short-term memory process where recognition of a familiar conspecific critically drives behavior toward the novel one (Kaidanovich-Beilin et al, 2011). Accordingly, the effects of mGluR5 and Oxtr knockdown observed in the lateral septum likely reflect disparate actions on social motivation versus social memory. In line with this view, the non-overlapping distribution of mGluR5 and Oxtr within separate lateral septal compartments suggests that in addition to different receptor types, different neuronal populations underlie the motivational versus cognitive aspects of social behavior. Given the tight relationship between the organization of septal neurons and their inputs, this further suggests that mGluR5- and Oxtr-positive cell populations are regulated by projections originating from different hippocampal subdivisions (Risold and Swanson, 1997) or other areas involved in the regulation of social behavior.

Our data on the distinct mechanisms of social behavioral phenotypes are supported by findings showing that mice containing NMDA receptors with reduced affinity for glycine have sociability deficits but normal preference for social novelty (Labrie et al, 2008). If confirmed in humans, these data suggest that treatment of social dysfunction in patients with mental disorders might require different approaches for individual components of social functioning. To target specifically the lateral septal receptor populations, potential mGluR5 and Oxtr-based therapeutics could take advantage of the rapid advances of technologies for guiding therapeutics to desired locations (Pilakka-Kanthikeel et al, 2013) or for activating defined neuronal circuits (Balconi and Canavesio, 2014).

\section{FUNDING AND DISCLOSURE}

The authors declare no conflict of interest.

\section{ACKNOWLEDGMENTS}

We thank the students Alexandra Garcia and Helen Chen for their help with the behavioral experiments and Dr Jian Xu for providing tissue of knockout mGluR5 mice for validation of the mGluR5 antibodies. This work was supported by NIMH Grants to JR (MH078064) and YFG (MH092065), Deutsche Forschungsemeinschaft fellowship to IM, and 'Integrated research on neuropsychiatric disorder' grant in the Strategic Research Program for Brain Sciences by the Ministry of Education, Culture, Sports, Science, and Technology of Japan (to KN).

\section{REFERENCES}

Balconi M, Canavesio Y (2014). High-frequency rTMS on DLPFC increases prosocial attitude in case of decision to support people. Soc Neurosci 9: 82-93.

Burket JA, Herndon AL, Winebarger EE, Jacome LF, Deutsch SI (2011). Complex effects of mGluR5 antagonism on sociability and stereotypic behaviors in mice: possible implications for the pharmacotherapy of autism spectrum disorders. Brain Res Bull 86: $152-158$.

Caldu X, Dreher JC (2007). Hormonal and genetic influences on processing reward and social information. Ann N Y Acad Sci 1118: $43-73$.

Caldwell HK (2012). Neurobiology of sociability. Adv Exp Med Biol 739: 187-205.

Carlson GC (2012). Glutamate receptor dysfunction and drug targets across models of autism spectrum disorders. Pharmacol Biochem Behav 100: 850-854.

Carter CS (1998). Neuroendocrine perspectives on social attachment and love. Psychoneuroendocrinology 23: 779-818.

Clifton NE, Morisot N, Girardon S, Millan MJ, Loiseau F (2013). Enhancement of social novelty discrimination by positive allosteric modulators at metabotropic glutamate 5 receptors: adolescent administration prevents adult-onset deficits induced by neonatal treatment with phencyclidine. Psychopharmacology (Berl) 225: 579-594.

Coghlan S, Horder J, Inkster B, Mendez MA, Murphy DG, Nutt DJ (2012). GABA system dysfunction in autism and related disorders: from synapse to symptoms. Neurosci Biobehav Rev 36: 2044-2055.

Cosford ND, Roppe J, Tehrani L, Schweiger EJ, Seiders TJ, Chaudary A, Rao S, Varney MA (2003). [ $\left.{ }^{3} \mathrm{H}\right]$-methoxymethylMTEP and $\left[{ }^{3} \mathrm{H}\right]$-methoxy-PEPy: potent and selective radioligands for the metabotropic glutamate subtype 5 (mGlu5) receptor. Bioorg Med Chem Lett 13: 351-354.

Crawley JN, Chen T, Puri A, Washburn R, Sullivan TL, Hill JM, Young NB, Nadler JJ, Moy SS, Young LJ, Caldwell HK, Young WS (2007). Social approach behaviors in oxytocin knockout mice: comparison of two independent lines tested in different laboratory environments. Neuropeptides 41: 145-163.

Crespi B, Badcock C (2008). Psychosis and autism as diametrical disorders of the social brain. Behav Brain Sci 31: 241-261.

de Vrij FM, Levenga J, van der Linde HC, Koekkoek SK, De Zeeuw CI, Nelson DL, Oostra BA, Willemsen R (2008). Rescue of behavioral phenotype and neuronal protrusion morphology in Fmr1 KO mice. Neurobiol Dis 31: 127-132.

Dolen G, Carpenter RL, Ocain TD, Bear MF (2010). Mechanismbased approaches to treating fragile X. Pharmacol Ther 127: 78-93.

Ferguson JN, Young LJ, Hearn EF, Matzuk MM, Insel TR, Winslow JT (2000). Social amnesia in mice lacking the oxytocin gene. Nat Genet 25: 284-288.

Gao C, Frausto SF, Guedea AL, Tronson NC, Jovasevic V, Leaderbrand K, Corcoran KA, Guzman YF, Swanson GT, Radulovic J (2011). IQGAP1 regulates NR2A signaling, spine density, and cognitive processes. J Neurosci 31: 8533-8542.

Gray J, McNaughton N (2000). The Neuropsychology of Anxiety. Oxford University Press: Oxford, UK.

Gunaydin LA, Grosenick L, Finkelstein JC, Kauvar IV, Fenno LE, Adhikari A, Lammel S, Mirzabekov JJ, Airan RD, Zalocusky KA, Tye KM, Anikeeva P, Malenka RC, Deisseroth K (2014). Natural neural projection dynamics underlying social behavior. Cell 157: $1535-1551$.

Guzman YF, Tronson NC, Jovasevic V, Sato K, Guedea AL, Mizukami H, Nishimori K, Radulovic J (2013). Fear-enhancing effects of septal oxytocin receptors. Nat Neurosci 16: 1185-1187.

Guzman YF, Tronson NC, Sato K, Mesic I, Guedea AL, Nishimori K, Radulovic J (2014). Role of oxytocin receptors in modulation of fear by social memory. Psychopharmacology (Berl) 231: 2097-2105.

Hitti FL, Siegelbaum SA (2014). The hippocampal CA2 region is essential for social memory. Nature 508: 88-92.

Insel TR, O'Brien DJ, Leckman JF (1999). Oxytocin, vasopressin, and autism: is there a connection? Biol Psychiatry 45: 145-157.

Kaidanovich-Beilin O, Lipina T, Vukobradovic I, Roder J, Woodgett JR (2011). Assessment of social interaction behaviors. J Vis Exp 48: 2473. 
Kiser D, Steemers B, Branchi I, Homberg JR (2012). The reciprocal interaction between serotonin and social behaviour. Neurosci Biobehav Rev 36: 786-798.

Koros E, Rosenbrock H, Birk G, Weiss C, Sams-Dodd F (2007). The selective mGlu5 receptor antagonist MTEP, similar to NMDA receptor antagonists, induces social isolation in rats. Neuropsychopharmacology 32: 562-576.

Labrie V, Lipina T, Roder JC (2008). Mice with reduced NMDA receptor glycine affinity model some of the negative and cognitive symptoms of schizophrenia. Psychopharmacology (Berl) 200: 217-230.

Lee HJ, Caldwell HK, Macbeth AH, Tolu SG, Young WS 3rd (2008). A conditional knockout mouse line of the oxytocin receptor. Endocrinology 149: 3256-3263.

Movsesyan VA, O'Leary DM, Fan L, Bao W, Mullins PG, Knoblach SM, Faden AI (2001). mGluR5 antagonists 2-methyl6-(phenylethynyl)-pyridine and (E)-2-methyl-6-(2-phenylethenyl)-pyridine reduce traumatic neuronal injury in vitro and in vivo by antagonizing $N$-methyl-D-aspartate receptors. J Pharmacol Exp Ther 296: 41-47.

Moy SS, Nadler JJ, Perez A, Barbaro RP, Johns JM, Magnuson TR, Piven J, Crawley JN (2004). Sociability and preference for social novelty in five inbred strains: an approach to assess autistic-like behavior in mice. Genes Brain Behav 3: 287-302.

Nakamoto M, Nalavadi V, Epstein MP, Narayanan U, Bassell GJ, Warren ST (2007). Fragile $X$ mental retardation protein deficiency leads to excessive mGluR5-dependent internalization of AMPA receptors. Proc Natl Acad Sci USA 104: 15537-15542.

Neumann ID (2008). Brain oxytocin: a key regulator of emotional and social behaviours in both females and males. J Neuroendocrinol 20: 858-865.

Nishimori K, Takayanagi Y, Yoshida M, Kasahara Y, Young LJ, Kawamata M (2008). New aspects of oxytocin receptor function revealed by knockout mice: sociosexual behaviour and control of energy balance. Prog Brain Res 170: 79-90.

O'Leary DM, Movsesyan V, Vicini S, Faden AI (2000). Selective mGluR5 antagonists MPEP and SIB-1893 decrease NMDA or glutamate-mediated neuronal toxicity through actions that reflect NMDA receptor antagonism. Br J Pharmacol 131: 1429-1437.

Panksepp J (1992). Oxytocin effects on emotional processes: separation distress, social bonding, and relationships to psychiatric disorders. Ann NY Acad Sci 652: 243-252.

Paxinos G, Franklin KB (2004). The Mouse Brain in Stereotaxic Coordinates. Gulf Profesional Publishing: San Diego.

Pilakka-Kanthikeel S, Atluri VS, Sagar V, Saxena SK, Nair M (2013). Targeted brain derived neurotropic factors (BDNF) delivery across the blood-brain barrier for neuro-protection using magnetic nano carriers: an in-vitro study. PLoS One 8: e62241.

Pobbe RL, Pearson BL, Defensor EB, Bolivar VJ, Young WS 3rd, Lee HJ, Blanchard DC, Blanchard RJ (2012). Oxytocin receptor knockout mice display deficits in the expression of autism-related behaviors. Horm Behav 61: 436-444.

Risold PY, Swanson LW (1997). Connections of the rat lateral septal complex. Brain Res Rev 24: 115-195.

Romano C, Sesma MA, McDonald CT, O'Malley K, Van den Pol AN, Olney JW (1995). Distribution of metabotropic glutamate receptor mGluR5 immunoreactivity in rat brain. J Comp Neurol 355: 455-469.

Ross HE, Young LJ (2009). Oxytocin and the neural mechanisms regulating social cognition and affiliative behavior. Front Neuroendocrinol 30: 534-547.

Sala M, Braida D, Donzelli A, Martucci R, Busnelli M, Bulgheroni E, Rubino T, Parolaro D, Nishimori K, Chini B (2013). Mice heterozygous for the oxytocin receptor gene $(\mathrm{Oxtr}(+/-))$ show impaired social behaviour but not increased aggression or cognitive inflexibility: evidence of a selective haploinsufficiency gene effect. J Neuroendocrinol 25: 107-118.

Sankoorikal GM, Kaercher KA, Boon CJ, Lee JK, Brodkin ES (2006). A mouse model system for genetic analysis of sociability: C57BL/ $6 \mathrm{~J}$ versus $\mathrm{BALB} / \mathrm{cJ}$ inbred mouse strains. Biol Psychiatry 59: 415-423.

Shigemoto R, Nomura S, Ohishi H, Sugihara H, Nakanishi S, Mizuno N (1993). Immunohistochemical localization of a metabotropic glutamate receptor, mGluR5, in the rat brain. Neurosci Lett 163: 53-57.

Silverman JL, Smith DG, Rizzo SJ, Karras MN, Turner SM, Tolu SS, Bryce DK, Smith DL, Fonseca K, Ring RH, Crawley JN (2012). Negative allosteric modulation of the mGluR5 receptor reduces repetitive behaviors and rescues social deficits in mouse models of autism. Sci Transl Med 4: 131-151.

Silverman JL, Tolu SS, Barkan CL, Crawley JN (2010). Repetitive self-grooming behavior in the BTBR mouse model of autism is blocked by the mGluR5 antagonist MPEP. Neuropsychopharmacology 35: 976-989.

Teng BL, Nonneman RJ, Agster KL, Nikolova VD, Davis TT, Riddick NV, Baker LK, Pedersen CA, Jarstfer MB, Moy SS (2013). Prosocial effects of oxytocin in two mouse models of autism spectrum disorders. Neuropharmacology 72: 187-196.

Thomas AM, Bui N, Graham D, Perkins JR, Yuva-Paylor LA, Paylor R (2011). Genetic reduction of group 1 metabotropic glutamate receptors alters select behaviors in a mouse model for fragile X syndrome. Behav Brain Res 223: 310-321.

Tronson NC, Guzman YF, Guedea AL, Huh KH, Gao C, Schwarz MK, Radulovic J (2010). Metabotropic glutamate receptor 5/Homer interactions underlie stress effects on fear. Biol Psychiatry 68: 1007-1015.

Tronson NC, Schrick C, Guzman YF, Huh KH, Srivastava DP, Penzes P, Guedea AL, Gao C, Radulovic J (2009). Segregated populations of hippocampal principal CA1 neurons mediating conditioning and extinction of contextual fear. J Neurosci 29: 3387-3394.

Won H, Lee HR, Gee HY, Mah W, Kim JI, Lee J, Ha S, Chung C, Jung ES, Cho YS, Park SG, Lee JS, Lee K, Kim D, Bae YC, Kaang BK, Lee MG, Kim E (2012). Autistic-like social behaviour in Shank2-mutant mice improved by restoring NMDA receptor function. Nature 486: 261-265.

Xu J, Zhu Y, Contractor A, Heinemann SF (2009). mGluR5 has a critical role in inhibitory learning. J Neurosci 29: 3676-3684.

Yan QJ, Rammal M, Tranfaglia M, Bauchwitz RP (2005). Suppression of two major Fragile $\mathrm{X}$ syndrome mouse model phenotypes by the mGluR5 antagonist MPEP. Neuropharmacology 49: 1053-1066.

Yang P, Chang CL (2014). Glutamate-mediated signaling and autism spectrum disorders: emerging treatment targets. Curr Pharm Des 20: 5186-5193.

Yap JJ, Covington HE III, Gale MC, Datta R, Miczek KA (2005). Behavioral sensitization due to social defeat stress in mice: antagonism at mGluR5 and NMDA receptors. Psychopharmacology (Berlin) 179: 230-239.

Yoshida M, Takayanagi Y, Inoue K, Kimura T, Young LJ, Onaka T, Nishimori K (2009). Evidence that oxytocin exerts anxiolytic effects via oxytocin receptor expressed in serotonergic neurons in mice. J Neurosci 29: 2259-2271. 